Kragujevac Journal of Mathematics

Volume 44(1) (2020), Pages 41-63.

\title{
ON THE TRANSMISSION-BASED GRAPH TOPOLOGICAL INDICES
}

\author{
R. SHARAFDINI ${ }^{1}$ AND T. RÉTI ${ }^{2}$
}

\begin{abstract}
The distance $d(u, v)$ between vertices $u$ and $v$ of a connected graph $G$ is equal to the number of edges in a minimal path connecting them. The transmission of a vertex $v$ is defined by $\sigma(v)=\sum_{u \in V(G)} d(v, u)$. A topological index is said to be a transmission-based topological index (TT index) if it includes the transmissions $\sigma(u)$ of vertices of $G$. Because $\sigma(u)$ can be derived from the distance matrix of $G$, it follows that transmission-based topological indices form a subset of distance-based topological indices. So far, relatively limited attention has been paid to TT indices, and very little systematic studies have been done. In this paper our aim was i) to define various types of transmission-based topological indices ii) establish lower and upper bounds for them, and iii) determine a family of graphs for which these bounds are best possible. Additionally, it has been shown in examples that using a group theoretical approach the transmission-based topological indices can be easily computed for a particular set of regular, vertex-transitive, and edge-transitive graphs. Finally, it is demonstrated that there exist TT indices which can be successfully applied to predict various physicochemical properties of different organic compounds. Some of them give better results and have a better discriminatory power than the most popular degree-based and distance-based indices (Randić, Wiener, Balaban indices).
\end{abstract}

\section{Introduction AND PRELIMINARIES}

Let $G$ be a simple connected graph with the finite vertex set $V(G)$ and the edge set $E(G)$, and denote by $n=|V(G)|$ and $m=|E(G)|$ the number of vertices and edges, respectively. Using the standard terminology in graph theory, we refer the reader to [44]. The degree $d(u)$ of the vertex $u \in V(G)$ is the number of the edges incident to $u$. The edge of the graph $G$ connecting the vertices $u$ and $v$ is denoted by $u v$.

Key words and phrases. Graph distance, topological index, transmission.

2010 Mathematics Subject Classification. Primary: 05C12. Secondary: 05C05, 05C07, 05 C90.

DOI 10.46793/KgJMat2001.041S

Received: July 29, 2017.

Accepted: February 14, 2018. 
The role of molecular descriptors (especially topological descriptors) is remarkable in mathematical chemistry especially in QSPR/QSAR investigations. In mathematical chemistry, the first Zagreb index $M_{1}(G)$ and the second Zagreb index $M_{2}(G)$ belong to the family of the most important degree-based molecular descriptors. They are defined as $[22,23,25,31,36]$

$$
M_{1}(G)=\sum_{u v \in E(G)} d(u)+d(v)=\sum_{u \in V(G)} d^{2}(u), \quad M_{2}(G)=\sum_{u v \in E(G)} d(u) d(v) .
$$

Similarly, the first variable Zagreb index and the second variable Zagreb index are defined as $[33,36,46]$

$$
M_{1}^{\lambda}(G)=\sum_{u \in V(G)} d(u)^{2 \lambda}, \quad M_{2}^{\lambda}(G)=\sum_{u v \in E(G)} d(u)^{\lambda} d(v)^{\lambda},
$$

where $\lambda$ is a real number.

The Randic index $R(G)$, the ordinary sum-connectivity index $X(G)$, the harmonic index $H(G)$ and geometric-arithmetic index $G A(G)$ are also widely used degree-based topological indices $[17,40,45,48-50]$. By definition,

$$
\begin{aligned}
& R(G)=\sum_{u v \in E(G)} \frac{1}{\sqrt{d(u) d(v)}}, \quad X(G)=\sum_{u v \in E(G)} \frac{1}{\sqrt{d(u)+d(v)}}, \\
& H(G)=\sum_{u v \in E(G)} \frac{2}{d(u)+d(v)}, \quad G A(G)=\sum_{u v \in E(G)} \frac{2 \sqrt{d(u) d(v)}}{d(u)+d(v)} .
\end{aligned}
$$

Let $\Delta=\Delta(G)$ and $\delta=\delta(G)$ be the maximum and the minimum degrees, respectively, of vertices of $G$. The average degree of $G$ is $\frac{2 m}{n}$. A connected graph $G$ is said to be bidegreed with degrees $\Delta$ and $\delta, \Delta>\delta \geq 1$, if at least one vertex of $G$ has degree $\Delta$ and at least one vertex has degree $\delta$, and if no vertex of $G$ has degree different from $\Delta$ or $\delta$. A connected bidegreed bipartite graph is called semi-regular if each vertex in the same part of a bipartition has the same degree. A graph $G$ is called regular if all its vertices have the same degree, otherwise it is said to be irregular. In many applications and problems in theoretical chemistry, it is important to know how a given graph is irregular. The (vertex) regularity of a graph is defined in several approaches. Two most frequently used graph topological indices that measure how irregular a graph is, are the irregularity and variance of degrees. Let $\operatorname{imb}(e)=|d(u)-d(v)|$ be the imbalance of an edge $e=u v \in E(G)$. In [1], the irregularity of $G$, which is a measure of irregularity of graph $G$, defined as

$$
\operatorname{irr}(G)=\sum_{e \in E(G)} \operatorname{imb}(e)=\sum_{u v \in E(G)}\left|d_{G}(u)-d_{G}(v)\right| .
$$

The variance of degrees of graph $G$ is defined as [7]

$$
\operatorname{Var}(G)=\frac{1}{n} \sum_{u \in V(G)}\left(d(u)-\frac{2 m}{n}\right)^{2}=\frac{M_{1}(G)}{n}-\frac{4 m^{2}}{n^{2}}
$$


Another measure of irregularity, which is called degree deviation, defined as [37]

$$
s(G)=\sum_{u \in V(G)}\left|d(u)-\frac{2 m}{n}\right| .
$$

It is worth mentioning that $\frac{s(G)}{n}$ is nothing but the mean deviation of the data set $(d(u) \mid u \in V(G))$.

The distance between the vertices $u$ and $v$ in graph $G$ is denoted by $d(u, v)$ and it is defined as the number of edges in a minimal path connecting them. The eccentricity $\varepsilon(v)$ of a vertex $v$ is the maximum distance from $v$ to any other vertex. The diameter $\operatorname{diam}(G)$ of $G$ is the maximum eccentricity among the vertices of $G$. The transmission (or status) of a vertex $v$ of $G$ is defined as $\sigma(v)=\sigma_{G}(v)=\sum_{u \in V(G)} d(v, u)$. A graph $G$ is said to be transmission regular [3] if $\sigma(u)=\sigma(v)$ for any vertex $u$ and $v$ of $G$. A transmission regular graph $G$ is called $k$-transmission regular if there exists a positive integer $k$, for which $\sigma(v)=k$ for any vertex $v$ of $G$. In $K_{n}$, the complete graph of order $n$, each vertex has transmission $n-1$. So it is $(n-1)$-transmission regular. The the cycle $C_{n}$ and the complete bipartite graph $K_{a, a}$ are transmission regular. It has been verified that there exist regular and non-regular transmission regular graphs [3]. Consider the polyhedron depicted in Figure 1. It is the rhombic dodecahedron that contains 14 vertices, (8 vertices of degree 3 and 6 vertices of degree 4), 24 edges and 12 faces, all of them are congruent rhombi. The graph $G_{R D}$ of the rhombic

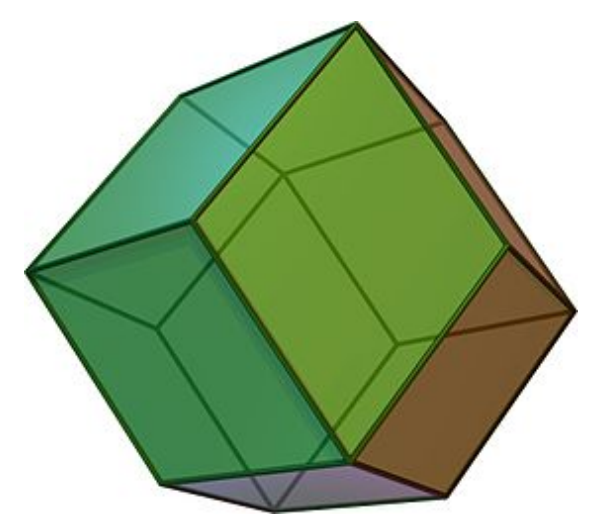

Figure 1. The rhombic dodecahedron

dodecahedron is a bidegreed, semi-regular 28-transmission regular graph (see Figure 2 ). An interesting observation is that the 14 -vertex polyhedral graph $G_{R D}$ depicted in Figure 2 is identical to the semi-regular graph published earlier in an alternative form in [3]. It is conjectured that $G_{R D}$ is the smallest non-regular, bipartite, polyhedral (3-connected) and transmission regular graph.

If $\omega$ is a vertex weight of graph $G$, then one can see that

$$
\sum_{\{u, v\} \subseteq V(G)}(\omega(u)+\omega(v)) d(u, v)=\sum_{v \in V(G)} \omega(v) \sigma(v) .
$$




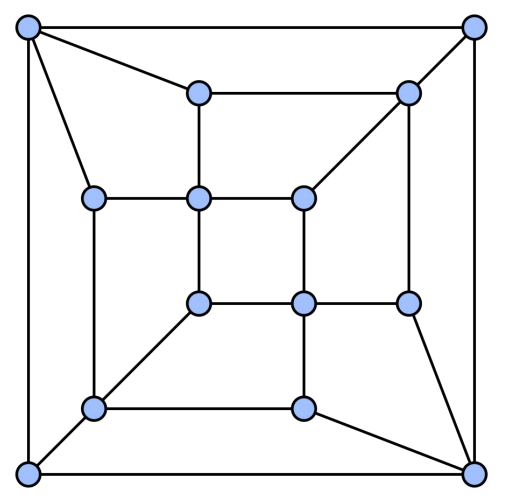

FiguRE 2. The edge-graph of the rhombic dodecahedron which is a 28-transmission regular graph but not regular

It is easy to construct various transmission-based indices having the same structure as the known degree-based topological indices. Based on this analogy-concept, the corresponding transmission-based indices are defined.

Let us define the transmission Randić index $R S(G)$, the transmission ordinary sum-connectivity index $X S(G)$, the transmission harmonic index $H S(G)$ and the transmission geometric-arithmetic index $G A S(G)$ as follows:

$$
\begin{gathered}
R S(G)=\sum_{u v \in E(G)} \frac{1}{\sqrt{\sigma(u) \sigma(v)}}, \quad X S(G)=\sum_{u v \in E(G)} \frac{1}{\sqrt{\sigma(u)+\sigma(v)}}, \\
H S(G)=\sum_{u v \in E(G)} \frac{2}{\sigma(u)+\sigma(v)}, \quad G A S(G)=\frac{n}{2 m} \sum_{u v \in E(G)} \frac{2 \sqrt{\sigma(u) \sigma(v)}}{\sigma(u)+\sigma(v)} .
\end{gathered}
$$

It follows that $G A S(G) \leq \frac{n}{2}$, with equality if and only if $G$ is a transmission regular graph.

The Wiener index $W(G)$, the Balaban index $J(G)$ and the sum-Balaban index $S J(G)$ represent a particular class of transmission-based topological indices. They are defined as $[4-6,9,10,16,21,51]$

$$
\begin{aligned}
W(G) & =\frac{1}{2} \sum_{u \in V G)} \sum_{v \in V(G)} d(u, v)=\frac{1}{2} \sum_{u \in V G)} \sigma(u), \\
J(G) & =\frac{m}{m-n+2} \sum_{u v \in E(G)} \frac{1}{\sqrt{\sigma(u) \sigma(v)}}=\frac{m}{m-n+2} R S(G), \\
S J(G) & =\frac{m}{m-n+2} \sum_{u v \in E(G)} \frac{1}{\sqrt{\sigma(u)+\sigma(v)}}=\frac{m}{m-n+2} X S(G) .
\end{aligned}
$$


In [39] the first transmission Zagreb index $M S_{1}(G)$ and the second transmission Zagreb index $M S_{2}(G)$ are defined as

$$
M S_{1}(G)=\sum_{u v \in E(G)} \sigma(u)+\sigma(v)=\sum_{u \in V(G)} d(u) \sigma(u), \quad M S_{2}(G)=\sum_{u v \in E(G)} \sigma(u) \sigma(v) .
$$

It is important to note that $M S_{1}(G)$ coincides with the degree distance $D D(G)$ that was introduced in [11,24] and [43].

In fact by (1.3),

$$
D D(G)=\sum_{\{u, v\} \subseteq V(G)}(d(u)+d(v)) d(u, v)=\sum_{v \in V(G)} d(v) \sigma(v)=M S_{1}(G) .
$$

Consequently, if $G$ is a $k$-transmission regular graph with $m$ vertices, then $D D(G)=$ $M S_{1}(G)=2 m k$.

Let us propose the variable degree transmission Zagreb index $M S D^{\lambda}(G)$ and the variable transmission Zagreb index $M S^{\lambda}(G)$ as follows

$$
M S D^{\lambda}(G)=\sum_{u \in V(G)} d(u) \sigma(u)^{2 \lambda-1}, \quad M S^{\lambda}(G)=\sum_{u \in V(G)} \sigma(u)^{2 \lambda},
$$

where $\lambda$ is a real number.

The eccentric distance sum of a graph $G$, denoted by $\xi^{d}(G)$, defined as [20]

$$
\xi^{d}(G)=\sum_{u \in V(G)} \varepsilon(u) \sigma(u)
$$

It follows from (1.3) that

$$
\xi^{d}(G)=\sum_{\{u, v\} \subseteq V(G)}(\varepsilon(u)+\varepsilon(v)) d(u, v)=\sum_{v \in V(G)} \varepsilon(v) \sigma(v) .
$$

Starting with (1.6) and (1.7) we introduce two transmission-based irregularity indices defined as follows. Let $G$ be a connected graph with $n$ vertices and $m$ edges. The transmission imbalance of an edge $e=u v \in E(G)$ is defined as $\operatorname{imb}_{\operatorname{Tr}}(e)=$ $\left|\sigma_{G}(u)-\sigma_{G}(v)\right|$. Let us define the transmission irregularity $\operatorname{irr}_{\operatorname{Tr}}(G)$ and the transmission variance $\operatorname{Var}_{\operatorname{Tr}}(G)$ of $G$ as follows:

$$
\begin{aligned}
\operatorname{irr}_{\operatorname{Tr}}(G) & =\sum_{e \in E(G)} \operatorname{imb}_{\operatorname{Tr}}(e)=\sum_{u v \in E(G)}\left|\sigma_{G}(u)-\sigma_{G}(v)\right| \\
\operatorname{Var}_{\operatorname{Tr}}(G) & =\frac{1}{n} \sum_{u \in V(G)}\left(\sigma_{G}(u)-\frac{2 W(G)}{n}\right)^{2}=\frac{1}{n} \sum_{u \in V(G)} \sigma_{G}(u)^{2}-\frac{4 W(G)^{2}}{n^{2}} \\
& =\frac{M S^{1}(G)}{n}-\frac{4 W(G)^{2}}{n^{2}} \geq 0
\end{aligned}
$$

where $\frac{2 W(G)}{n}$ is the average vertex transmission of $G$. It is obvious that $\operatorname{Var}_{\operatorname{Tr}}(G)$ is equal to zero if and only if $G$ is transmission regular. 
Let us also define the transmission-based topological indices $Q S_{e}(G)$ and $Q S_{v, e}(G)$ as follows

$$
Q S_{e}(G)=\frac{1}{m} \operatorname{irr}_{\operatorname{Tr}}(G), \quad Q S_{v, e}(G)=\frac{n}{2}\left(1+\frac{1}{m} \operatorname{irr}_{\operatorname{Tr}}(G)\right)=\frac{n}{2}\left(1+Q S_{e}(G)\right) .
$$

Remark 1.1. Let $G$ be an $n$-vertex graph. Comparing topological indices $G A S(G)$ and $Q S_{v, e}(G)$, we get

$$
G A S(G) \leq \frac{n}{2} \leq Q S_{v, e}(G)
$$

Equalities hold in both sides simultaneously if and only if $G$ is transmission regular.

\section{Establishing Lower And Upper Bounds}

Lemma 2.1. Let $G$ be a connected graph with $n \geq 2$ vertices and $m$ edges. Then

$$
\begin{aligned}
& 0 \leq \operatorname{irr}_{\operatorname{Tr}}(G) \leq m(n-2), \\
& 0 \leq \sum_{u v \in E(G)}(\sigma(u)-\sigma(v))^{2} \leq m(n-2)^{2} .
\end{aligned}
$$

The equality on the right-hand sides holds if and only if $G$ is isomorphic to $S_{n}$. The equality on the left-hand sides holds if and only if $G$ is transmission regular.

Proof. For an arbitrary edge $u v$ of $G$, we have $|\sigma(u)-\sigma(v)| \leq n-2$. Therefore,

$$
\operatorname{irr}_{\operatorname{Tr}}(G)=\sum_{u v \in E(G)}|\sigma(u)-\sigma(v)| \leq \sum_{u v \in E(G)}(n-2)=m(n-2) .
$$

It is trivial that in both formulas the equality on the right-hand side holds if and only if $G$ isomorphic to $S_{n}$, since the star is the only graph where equality holds for each edge.

Corollary 2.1. Let $T$ be a tree with $n \geq 2$ vertices. Then

$$
\begin{aligned}
& 0 \leq \operatorname{irr}_{\operatorname{Tr}}(T) \leq(n-1)(n-2), \\
& 0 \leq \sum_{u v \in E(T)}(\sigma(u)-\sigma(v))^{2} \leq(n-1)(n-2)^{2} .
\end{aligned}
$$

The equality on the right-hand sides holds if and only if $G$ is isomorphic to $S_{n}$. The equality on the left-hand sides holds if and only if $G$ is transmission regular.

Proof. It is a consequence of Lemma 2.1 and the fact that a tree with $n$ vertices has exactly $n-1$ edges.

Corollary 2.2. Let $G$ be a connected graph with $n \geq 2$ vertices. Then

$$
(n-2) \geq Q S_{e}(G) \geq 0
$$

and

$$
\frac{n(n-1)}{2} \geq Q S_{v, e}(G) \geq \frac{n}{2}
$$

The upper bounds are achieved if and only if $G$ is isomorphic to $S_{n}$ and the lower bounds are achieved if and only if $G$ is transmission regular. 
Proof. It is a direct consequence of Lemma 2.1.

Lemma 2.2. Let $G$ be a connected graph with $n \geq 3$ vertices and with maximum vertex degree $\Delta$. Then for each arbitrary vertex $u$ of $G$

$$
\sigma(u) \geqslant 2(n-1)-d(u) \geqslant 2(n-1)-\Delta \geqslant n-1 .
$$

Proof. Because $n-1 \geq \Delta \geq d(u)$ one obtains that

$$
\begin{aligned}
\sigma(u) & =\sum_{(w \in V \mid d(u, w)=1)} d(u, w)+\sum_{(w \in V \mid d(u, w)>1)} d(u, w)=d(u)+\sum_{(w \in V \mid d(u, w)>1)} d(u, w) \\
& \geqslant d(u)+2(n-1-d(u))=2 n-2-d(u) \geqslant 2(n-1)-\Delta \geqslant n-1 .
\end{aligned}
$$

Remark 2.1. There are several graphs containing a vertex $u$ for which $\sigma(u)=n-1$. For example, $\sigma(u)=d(u)=n-1$ for any vertex $u$ of a complete graph $K_{n}$.

Remark 2.2. Let $G$ be a connected graph. It is easy to see that for any $u \in V(G)$, $\sigma(u) \geqslant 2(n-1)-d(u)$, with equality if and only if $\varepsilon(u) \leq 2$. This implies that

(i) $\sigma(u)=2(n-1)-d(u)$ for any vertex $u$ of a connected graph $G$ if and only if $\operatorname{diam}(G) \leq 2$

(ii) if $G$ is a connected graph with $\operatorname{diam}(G) \leq 2$, then $G$ is transmission regular if and only if $G$ is regular.

Proposition 2.1. Let $G$ be a connected graph with $n$ vertices. Then

$$
M S D^{\frac{3}{2}}(G) \geq 2(n-1) M S^{1}(G)-M S^{\frac{3}{2}}(G),
$$

with equality if and only if $\operatorname{diam}(G) \leq 2$.

Proof. It follows from Lemma 2.2 that

$\sum_{u \in V(G)} d(u) \sigma^{2}(u) \geq \sum_{u \in V(G)}(2 n-2-\sigma(u)) \sigma^{2}(u)=2(n-1) \sum_{u \in V(G)} \sigma^{2}(u)-\sum_{u \in V(G)} \sigma^{3}(u)$,

and by Remark 2.2, the equality holds if and only if $\operatorname{diam}(G) \leq 2$.

Proposition 2.2. Let $G$ be a connected graph with $n$ vertices. Then

$$
M S_{1}(G) \geq 4(n-1) W(G)-M S^{1}(G),
$$

with equality if and only if $\operatorname{diam}(G) \leq 2$.

Proof. It follows from Lemma 2.2 that

$\sum_{u \in V(G)} d(u) \sigma(u) \geq \sum_{u \in V(G)}(2 n-2-\sigma(u)) \sigma(u)=2(n-1) \sum_{u \in V(G)} \sigma(u)-\sum_{u \in V(G)} \sigma^{2}(u)$.

It follows from Remark 2.2 that the equality holds if and only if $\operatorname{diam}(G) \leq 2$.

Lemma 2.3. Let $G$ be a connected graph with $n$ vertices and $m$ edges. If $\operatorname{diam}(G) \leq 2$, then

(i) $\operatorname{irr}_{\operatorname{Tr}}(G)=\operatorname{irr}(G) \geq 0$;

(ii) $Q S_{v, e}(G)=\frac{n}{2}\left(1+\frac{1}{m} \operatorname{irr}(G)\right) \geq \frac{n}{2}$. 
In particular, in both cases equality holds if and only if $G$ is regular.

Proof. $\quad$ (i) It is a direct consequence of Lemma 2.1 and Remark 2.2.

(ii) It follows directly from part (i).

Corollary 2.3. Let $K_{p, q}$ be the complete bipartite graph with $p+q$ vertices and with parts of size $p$ and $q$. Then

(i) $\operatorname{irr}_{\operatorname{Tr}}\left(K_{p, q}\right)=p q|p-q| \geq 0$;

(ii) $Q S_{v, e}\left(K_{p, q}\right)=\frac{p+q}{2}(1+|p-q|) \geq \frac{p+q}{2}$, specially $Q S_{v, e}\left(S_{n}\right)=\frac{n(n-1)}{2}$.

In particular, the equalities in (i) and (ii) hold if and only if $p=q$.

Proof. $\quad$ (i) $\operatorname{Since} \operatorname{diam}\left(K_{p, q}\right)=2$ and $\left|E\left(K_{p, q}\right)\right|=p q$, it follows from Lemma 2.3 (i) that $\operatorname{irr}_{\operatorname{Tr}}\left(K_{p, q}\right)=\operatorname{irr}\left(K_{p, q}\right)=\sum_{u v \in E\left(K_{p, q}\right)}|p-q|=p q|p-q|$.

(ii) Since $\operatorname{diam}\left(K_{p, q}\right)=2$ and $\left|V\left(K_{p, q}\right)\right|=p+q$, it follows from Lemma 2.3 (ii) that

$$
Q S_{v, e}\left(K_{p, q}\right)=\frac{p+q}{2}(1+|p-q|) \geq \frac{p+q}{2} .
$$

Specially, let $n \geq 2$ and $p=1$ and $q=n-1$. Then $K_{p, q}$ is isomorphic to the star $S_{n},(n=p+q)$. Consequently, we obtain that

$$
Q S_{v, e}\left(S_{n}\right)=\frac{n}{2}(1+|2-n|)=\frac{n(n-1)}{2} .
$$

It follows from Lemma 2.3 that the equalities in (i) and (ii) hold if and only if $K_{p, q}$ is regular if and only if $p=q$.

An edge $u v$ of a connected graph $G$ is said to be a strong edge of $G$, if $|d(u)-d(v)|>$ 0 . Denote by $\operatorname{es}(G)$ the number of strong edges of $G$. It is obvious that if $G$ is a connected graphs, then $e s(G)=0$ if and only if $G$ is regular. From this observation it follows that the topological invariant es $(G)$ can be considered as a graph irregularity index. There are several graphs in which each edge is strong, that is $e s(G)=|E(G)|$. For example, es $\left(K_{p, q}\right)=\left|E\left(K_{p, q}\right)\right|=p q$ if $p$ is not equal to $q$. It can be easily constructed a tree graph $T$ with an arbitrary large edge number $m(T)$, for which $e s(T)=m(T)$. Consider the $(n \geq 5)$-vertex windmill graph denoted by $W d(n)$. It is a graph with diameter 2 , with the vertex number $n=2 k+1$ and with the edge number $m=3 k$, where $k \geq 2$ is an arbitrary positive integer. Note that $e s(W d(n))=2 k=\frac{2}{3} m=n-1$.

Proposition 2.3. For the windmill graph $W d(n)$ we have

(i) $\operatorname{irr}_{\operatorname{Tr}}(W d(n))=e s(W d(n))(n-3)=\frac{2}{3} m(n-3)=(n-1)(n-3)$;

(ii) $Q S_{v, e}(W d(n))=\frac{n}{2}\left(1+\frac{2}{3}(n-3)\right)$.

Proof. (i) Let $E_{0}$ be the set of strong edges of $W d(n)$. It is easy to see that

$$
E_{0}=\{u v \in E(W d(n)) \mid d(u)=2, d(v)=n-1\}, \quad \text { es }(W d(n))=\left|E_{0}\right| .
$$


Since $\operatorname{diam}(W d(n))=2$, it follows from Lemma 2.3 (i) that

$$
\begin{aligned}
\operatorname{irr}_{\operatorname{Tr}}(W d(n))=\operatorname{irr}(W d(n)) & =\sum_{u v \in E_{0}}|d(u)-d(v)|=\sum_{u v \in E_{0}}|2-(n-1)| \\
& =e s(W d(n))|2-(n-1)| \\
& =\frac{2}{3} m(n-3)=(n-1)(n-3) .
\end{aligned}
$$

(ii) It follows from part (i) that

$$
\begin{aligned}
Q S_{v, e}(W d(n)) & =\frac{n}{2}\left(1+\frac{1}{m} \operatorname{irr}_{\operatorname{Tr}}(W d(n))\right)=\frac{n}{2}\left(1+\frac{2}{3}(n-3)\right) \\
& =\frac{n}{2}\left(1+\frac{1}{m}(n-1)(n-3)\right) .
\end{aligned}
$$

Lemma 2.4. [32] Let $P_{n}$ be a path of order $n$ such that $V\left(P_{n}\right)=\left\{v_{0}, v_{1}, \ldots, v_{n-1}\right\}$ and $E\left(P_{n}\right)=\left\{v_{i} v_{i+1} \mid i=0, \ldots, n-2\right\}$. Then for each $0 \leq i \leq n-1$

$$
\sigma_{P_{n}}\left(v_{i}\right)=\frac{1}{2}\left(2 i^{2}-2(n-1) i+(n-1)^{2}+(n-1)\right) .
$$

The following is a direct consequence of Lemma 2.4 .

Proposition 2.4. The transmission irregularity index of $P_{n}$ is given by

$$
\operatorname{irr}_{\operatorname{Tr}}\left(P_{n}\right)= \begin{cases}\frac{n(n-2)}{2}, & \text { if } n \text { is even } \\ \frac{(n-1)^{2}}{2}, & \text { if } n \text { is odd. }\end{cases}
$$

For an edge $u v$ of a connected graph $G$, define the positive integers $N_{u}$ and $N_{v}$ where $N_{u}$ is the number of vertices of $G$ whose distance to vertex $u$ is smaller than distance to vertex $v$, and analogously, $N_{v}$ is the number of vertices of $G$ whose distance to the vertex $v$ is smaller than to $u$. The number of vertices equidistant from $u$ and $v$ is denoted by $N_{u v}$. An edge $u v$ of $G$ is called a distance-balanced edge if $N_{u}=N_{v}$. A graph $G$ is said to be distance-balanced [26] if its each edge is distance-balanced. It is known that a connected graph $G$ is transmission regular if and only if $G$ is distance balanced $[3,26]$.

The Szeged index $S z(G)$ and the revised Szeged index $S z^{*}(G)$ of a connected graph $G$ are defined as $[29,35,38]$

$$
S z(G)=\sum_{u v \in E(G)} N_{u} N_{v}, \quad S z^{*}(G)=\sum_{u v \in E(G)}\left(N_{u}+\frac{N_{u v}}{2}\right)\left(N_{v}+\frac{N_{u v}}{2}\right) .
$$

Remark 2.3. For any connected graph $G$ with $n$ vertices, the following known relations are fulfilled $[3,12,13,16,28,29,35,38,47]$.

(i) For any edge $u v$ of $G, n=N_{u}+N_{v}+N_{u v}$. This implies that a graph $G$ is bipartite if and only if $n=N_{u}+N_{v}$ holds for any edge $u v$ of $G$.

(ii) The inequality $S z(G) \geq W(G)$ is fulfilled.

(iii) $S z(G) \leq S z^{*}(G)$ with equality if and only if $G$ is bipartite. 
(iv) For an $n$-vertex tree $T, W\left(S_{n}\right) \leq W(T) \leq W\left(P_{n}\right)$.

(v) For a tree graph $T, S z^{*}(T)=S z(T)=W(T)$.

The fundamental properties of Wiener index and their extremal graphs are summarized in $[9,12,13,16,21]$. Transmission regular graphs are characterized by the following property.

Lemma 2.5. $[3,26,29]$ Let $G$ be a connected graph with $n$ vertices and $m$ edges. Then

$$
S z^{*}(G) \leq \frac{n^{2} m}{4}
$$

with equality if and only if $G$ is transmission regular.

Lemma 2.6 ([3,12]). Let $G$ be a connected graph and let uv be an edge of $G$. Then

$$
\sigma(u)-\sigma(v)=N_{v}-N_{u} .
$$

Lemma 2.7. Let $G$ be a connected graph. Then the following hold:
(i) $\operatorname{irr}_{\operatorname{Tr}}(G)=\sum_{u v \in E(G)}\left|N_{u}-N_{v}\right| \geq 0$;
(ii) $\sum_{u v \in E(G)}\left(N_{u}-N_{v}\right)^{2}=M S D^{\frac{3}{2}}(G)-2 M S_{2}(G) \geq 0$;
(iii) $\sum_{u v \in E(G)}(\sigma(u)-\sigma(v))^{2}=\sum_{u v \in E(G)}\left(N_{u}^{2}+N_{v}^{2}\right)-2 S z(G) \geq 0$;
(iv) $\sum_{u v \in E(G)}\left(N_{u}^{2}+N_{v}^{2}\right)=M S D^{\frac{3}{2}}(G)+2 S z(G)-2 M S_{2}(G)$.

In (i), (ii), and (iii) the equality holds if and only if $G$ is transmission regular.

Proof. $\quad$ (i) This is a direct consequence of Lemma 2.6.

(ii)

$$
\begin{aligned}
0 \leq \sum_{u v \in E(G)}\left(N_{u}-N_{v}\right)^{2} & =\sum_{u v \in E(G)}(\sigma(u)-\sigma(v))^{2} \\
& =\sum_{u v \in E(G)}\left(\sigma^{2}(u)+\sigma^{2}(v)\right)-2 \sum_{u v \in E(G)} \sigma(u) \sigma(v) \\
& =\sum_{u \in V(G)} d(u) \sigma^{2}(u)-2 M S_{2}(G) \\
& =M S D^{\frac{3}{2}}(G)-2 M S_{2}(G) .
\end{aligned}
$$

(iii)

$$
\begin{aligned}
0 \leq \sum_{u v \in E(G)}(\sigma(u)-\sigma(v))^{2} & =\sum_{u v \in E(G)}\left(N_{u}-N_{v}\right)^{2} \\
& =\sum_{u v \in E(G)}\left(N_{u}^{2}+N_{v}^{2}\right)-2 S z(G) .
\end{aligned}
$$


(iv) It follows from the proof of the part (ii) and (iii) that

$$
\begin{aligned}
\sum_{u v \in E(G)}\left(N_{u}^{2}+N_{v}^{2}\right) & =\sum_{u v \in E(G)}(\sigma(u)-\sigma(v))^{2}+2 S z(G) \\
& =M S D^{\frac{3}{2}}(G)-2 M S_{2}(G)+2 S z(G) .
\end{aligned}
$$

Remark 2.4. Based on Lemma 2.7, the transmission-based topological index $Q S_{v, e}(G)$ can be represented in the following alternative form:

$$
Q S_{v, e}(G)=\frac{n}{2}\left(1+\frac{1}{m} \sum_{u v \in E(G)}|\sigma(u)-\sigma(v)|\right)=\frac{n}{2}\left(1+\frac{1}{m} \sum_{u v \in E(G)}\left|N_{u}-N_{v}\right|\right) .
$$

Proposition 2.5. Let $G$ be a connected graph with $n$ vertices and $m$ edges. Then

$$
n^{2} m \geq M S D^{\frac{3}{2}}(G)+4 S z(G)-2 M S_{2}(G),
$$

with equality if and only if $G$ is a bipartite graph.

Proof. Let $G$ be a connected graph with $n$ vertices. It follows from Remark 2.3 (i) that for any edge $u v$ of $G, N_{u}+N_{v} \leq n$, with equality if and only if $G$ is bipartite. This implies that

$$
n^{2} \geq\left(N_{u}+N_{v}\right)^{2}=\left(N_{u}^{2}+N_{v}^{2}\right)+2 N_{u} N_{v},
$$

with equality if and only if $G$ is bipartite. Consequently, by Lemma 2.7 (iv) we have

$$
\begin{aligned}
n^{2} m=\sum_{u v \in E(G)} n^{2} & \geq \sum_{u v \in E(G)}\left(N_{u}^{2}+N_{v}^{2}\right)+2 \sum_{u v \in E(G)} N_{u} N_{v} \\
& =\sum_{u v \in E(G)}\left(N_{u}^{2}+N_{v}^{2}\right)+2 S z(G) \\
& =M S D^{\frac{3}{2}}(G)+4 S z(G)-2 M S_{2}(G),
\end{aligned}
$$

with equality if and only if $G$ is bipartite.

Proposition 2.6. Let $G$ be a connected graph with $n$ vertices. Then

$$
\operatorname{irr}_{\operatorname{Tr}}(G)=\sum_{u v \in E(G)}\left|N_{u}-N_{v}\right| \geq \frac{1}{n} \sum_{u v \in E(G)}\left|N_{u}^{2}-N_{v}^{2}\right|
$$

with equality if and only if $G$ is a bipartite graph.

Proof. Let $G$ be a connected graph with $n$ vertices. It follows from Remark 2.3 (i) that for any edge $u v$ of $G, N_{u}+N_{v} \leq n$, with equality if and only if $G$ is bipartite. Therefore, it follows from Lemma 2.6 and

$$
\left|N_{u}^{2}-N_{v}^{2}\right|=\left(N_{u}+N_{v}\right)\left|N_{u}-N_{v}\right| \leq n\left|N_{u}-N_{v}\right|=n|\sigma(u)-\sigma(v)|,
$$

with equality if and only if $G$ is bipartite. This implies that (2.1) holds with equality if and only if $G$ is bipartite. 
Corollary 2.4. Let $T_{n}$ be an $n$ vertex tree. Then

$$
\begin{aligned}
M S_{2}\left(T_{n}\right) & =2 W\left(T_{n}\right)+\frac{1}{2} M S D^{\frac{3}{2}}\left(T_{n}\right)-\frac{n^{2}(n-1)}{2}, \\
\operatorname{irr}_{\operatorname{Tr}}\left(T_{n}\right) & =\frac{1}{n} \sum_{u v \in E\left(T_{n}\right)}\left|N_{u}^{2}-N_{v}^{2}\right| .
\end{aligned}
$$

Proof. It is a consequence of Proposition 2.5, Proposition 2.6 and Remark 2.3, since a tree with $n$ vertices is bipartite and has exactly $n-1$ edges.

Proposition 2.7. [12] Let $G_{B}$ be a connected bipartite graph with $n$ vertices and $m$ edges. Then

$$
S z^{*}\left(G_{B}\right)=S z\left(G_{B}\right)=\frac{n^{2} m}{4}-\frac{1}{4} \sum_{u v \in E\left(G_{B}\right)}(\sigma(u)-\sigma(v))^{2} \leq \frac{n^{2} m}{4},
$$

with equality if and only if $G$ is transmission regular.

Corollary 2.5. Let $G_{B}$ be a connected bipartite graph with $n$ vertices and $m$ edges. Then

$$
Q S_{v, e}\left(G_{B}\right) \leq \sqrt{n^{2}-\frac{4}{m} S z\left(G_{B}\right)},
$$

with equality if and only if $|\sigma(u)-\sigma(v)|$ is constant for any edge $u v \in G_{B}$.

Proof. Using Cauchy-Schwartz inequality and Proposition 2.7 one obtains for $G_{B}$ that

$$
\left(\frac{1}{m} \sum_{u v \in E\left(G_{B}\right)}|\sigma(u)-\sigma(v)|\right)^{2} \leq \frac{1}{m} \sum_{u v \in E\left(G_{B}\right)}(\sigma(u)-\sigma(v))^{2}=n^{2}-\frac{4}{m} S z\left(G_{B}\right),
$$

with equality if and only if $|\sigma(u)-\sigma(v)|$ is constant for any edge $u v \in G_{B}$. Consequently,

$$
\frac{1}{m} \sum_{u v \in E\left(G_{B}\right)}|\sigma(u)-\sigma(v)| \leq \sqrt{n^{2}-\frac{4}{m} S z\left(G_{B}\right)},
$$

with equality if and only if $|\sigma(u)-\sigma(v)|$ is constant for any edge $u v \in G_{B}$. Because

$$
Q S_{v, e}\left(G_{B}\right)-\frac{n}{2}=\frac{n}{2 m} \sum_{u v \in E\left(G_{B}\right)}|\sigma(u)-\sigma(v)|
$$

we have

$$
Q S_{v, e}\left(G_{B}\right)-\frac{n}{2} \leq \frac{n}{2} \sqrt{n^{2}-\frac{4}{m} S z\left(G_{B}\right)},
$$

with equality if and only if $|\sigma(u)-\sigma(v)|$ is constant for any edge $u v \in G_{B}$.

Lemma 2.8. [12] Let $T_{n}$ be an n-vertex tree. Then

$$
S z\left(T_{n}\right)=W\left(T_{n}\right)=\frac{1}{4}\left(n(n-1)+M S_{1}\left(T_{n}\right)\right) .
$$


The following proposition demonstrates that the Wiener index and the first transmission Zagreb index are closely related.

Proposition 2.8. Let $T_{n}$ be an n-vertex tree. Then

$$
M S_{1}\left(T_{n}\right)=4 W\left(T_{n}\right)-n(n-1)=4 S z\left(T_{n}\right)-n(n-1) .
$$

Proof. For any connected graph $G$ we have

$$
M S_{1}(G)=\sum_{u v \in E(G)}(\sigma(u)+\sigma(v))=\sum_{u \in V(G)} d(u) \sigma(u) .
$$

Therefore, by Lemma 2.8 the result follows.

Remark 2.5. As a consequence of (2.2), we conclude that in the family of $n$-vertex trees there is a linear correspondence (a perfect linear correlation) between the topological indices $W\left(T_{n}\right)$ and $M S_{1}\left(T_{n}\right)$.

In [39] it is reported that for a connected graph $G, W(G)<M S_{1}(G)$. This relation can be strengthened as follows.

Proposition 2.9. Let $G$ be a connected graph with minimum degree $\delta$ and maximum degree $\Delta$. Then

$$
2 \delta W(G) \leq M S_{1}(G) \leq 2 \Delta W(G)
$$

and equalities hold in both sides if and only if $G$ is a regular graph.

Proof. Because for any connected graph $G, M S_{1}(G)=\sum_{u v \in E(G)}(\sigma(u)+\sigma(v))=$ $\sum_{u \in V(G)} d(u) \sigma(u)$, and for any vertex $u$ of $G, \delta \leq d(u) \leq \Delta$, we have that

$$
\left.2 \delta W G) \leq \sum_{u v \in E(G)}(\sigma(u)+\sigma(v))=\sum_{u \in V(G)} d(u) \sigma(u) \leq 2 \Delta W G\right) .
$$

Consequently, if $G$ is an $r$-regular graph, we have $M S_{1}(G)=2 r W(G)$.

Corollary 2.6. Let $T_{n}$ be an $n$-vertex tree. Then

$$
(n-1)(3 n-4) \leq M S_{1}\left(T_{n}\right) \leq \frac{1}{3} n(n-1)(2 n-1),
$$

where

(i) the right-hand side equality holds if and only if $T_{n}$ is the path $P_{n}$;

(ii) the left-hand side equality holds if and only if $T_{n}$ is the star $S_{n}$.

Proof. For an $n$-vertex tree $T_{n}$ we have $W\left(S_{n}\right) \leq W\left(T_{n}\right) \leq W\left(P_{n}\right)$, where $W\left(S_{n}\right)=$ $(n-1)^{2}$ and $W\left(P_{n}\right)=\frac{\left(n^{3}-n\right)}{6}$. Therefore, from Proposition 2.8, we have the following inequalities:

$$
M S_{1}\left(T_{n}\right) \leq \frac{4 n(n-1)(n+1)}{6}-n(n-1)=\frac{1}{3} n(n-1)(2 n-1),
$$

with equality if and only if $T_{n}$ is the path $P_{n}$, and

$$
M S_{1}\left(T_{n}\right) \geq 4(n-1)^{2}-n(n-1)=(n-1)(3 n-4),
$$

with equality if and only if $T_{n}$ is the star $S_{n}$. 
The following is a direct consequence of Proposition 2.9.

Corollary 2.7. If $G_{b e}$ is a benzenoid graph with $\Delta=3$ and $\delta=2$, then

$$
4 W\left(G_{b e}\right) \leq M S_{1}\left(G_{b e}\right) \leq 6 W\left(G_{b e}\right) .
$$

It is easy to show that the inequality represented by

$$
M S_{2}(G)=\sum_{u v \in E(G)} \sigma(u) \sigma(v) \leq \frac{1}{2} M S D^{\frac{3}{2}}(G),
$$

can be sharpened in the following form.

Proposition 2.10. Let $G$ be a connected graph with $m$ edges. Then

$$
M S_{2}(G) \leq \frac{1}{2} M S D^{\frac{3}{2}}(G)-\frac{1}{2 m} \operatorname{irr}_{\operatorname{Tr}}(G)^{2},
$$

with equality if and only if $|\sigma(u)-\sigma(v)|$ is constant for any $u v \in E(G)$.

Proof. Using Cauchy-Schwartz inequality we have

$$
\begin{aligned}
\left(\frac{1}{m} \sum_{u v \in E(G)}|\sigma(u)-\sigma(v)|\right)^{2} & \leq \frac{1}{m} \sum_{u v \in E(G)}(\sigma(u)-\sigma(v))^{2} \\
& =\frac{1}{m} \sum_{u v \in E(G)}\left(\sigma^{2}(u)+\sigma^{2}(v)\right)-\frac{2}{m} \sum_{u v \in E(G)} \sigma(u) \sigma(v),
\end{aligned}
$$

with equality if and only if $|\sigma(u)-\sigma(v)|$ is constant for any $u v \in E(G)$. It follows that

$$
M S_{2}(G) \leq \frac{1}{2} M S D^{\frac{3}{2}}(G)-\frac{1}{2 m} \operatorname{irr}_{\operatorname{Tr}}(G)^{2}
$$

with equality if and only if $|\sigma(u)-\sigma(v)|$ is constant for any $u v \in E(G)$.

Corollary 2.8. Let $G$ be a connected graph with $m$ edges. If $\operatorname{diam}(G) \leq 2$, then

$$
M S_{2}(G) \leq \frac{1}{2} M S D^{\frac{3}{2}}(G)-\frac{1}{2 m} \operatorname{irr}(G)^{2},
$$

with equality if and only if $|d(u)-d(v)|$ is constant for any uv $\in E(G)$.

Proof. Let $G$ be a connected graph with $m$ edges. It follows from Remark 2.2 that for any $u v \in E(G),|d(u)-d(v)|$ is constant if $\operatorname{diam}(G) \leq 2$. Now the result follows from Lemma 2.3 and Proposition 2.10.

Lemma 2.9. [14,43] Let $G$ be a connected graph with $n$ vertices and $m$ edges. Then

$$
W(G) \geq n(n-1)-m,
$$

with equality if and only if $\operatorname{diam}(G) \leq 2$. (For example, the equality holds for complete graphs, complete bipartite and complete multipartite graphs, moreover wheel graphs and windmill graphs composed of triangles.) 
Proposition 2.11. Let $G$ be a connected $k$-transmission regular with $n$ vertices and $m$ edges. Then

$$
k=\frac{2 W(G)}{n} \geq 2(n-1)-\frac{2 m}{n},
$$

with equality if and only if $\operatorname{diam}(G) \leq 2$.

Proof. Since $G$ is $k$-transmission regular, $W(G)=\frac{n k}{2}$ holds. Now the result follows from Lemma 2.9.

Proposition 2.12. Let $G$ be a connected graph with $n$ vertices and $m$ edges. Then

$$
M S^{1}(G) \geq 4(n-1) W(G)-M S_{1}(G) \geq 4(n-1)\left(n^{2}-n-m\right)-M S_{1}(G),
$$

and equalities hold in both sides simultaneously if $\operatorname{diam}(G) \leq 2$.

Proof. The result follows directly, using Lemma 2.9 and Proposition 2.2.

Proposition 2.13. Let $G$ be a connected graph with $n$ vertices and $m$ edges. Then

$$
M S_{1}(G) \leq \sqrt{m\left(M S D^{\frac{3}{2}}(G)+2 M S_{2}(G)\right)}
$$

with equality if and only if $\sigma(u)+\sigma(v)$ is constant for each edge uv $\in E(G)$.

Proof. Using the Cauchy-Schwartz inequality, we obtain

$$
\begin{aligned}
\left(\frac{1}{m} \sum_{u v \in E(G)}(\sigma(u)+\sigma(v))\right)^{2} & \leq \frac{1}{m} \sum_{u v \in E(G)}(\sigma(u)+\sigma(v))^{2} \\
& =\frac{1}{m}\left(\sum_{u v \in E(G)}\left(\sigma^{2}(u)+\sigma^{2}(v)\right)+2 \sum_{u v \in E(G)} \sigma(u) \sigma(v)\right),
\end{aligned}
$$

with equality if and only if $\sigma(u)+\sigma(v)$ is constant for each edge $u v \in E(G)$. This implies that

$$
\left(\frac{1}{m} M S_{1}(G)\right)^{2} \leq \frac{1}{m}\left(M S D^{\frac{3}{2}}(G)+2 M S_{2}(G)\right)
$$

with equality if and only if $\sigma(u)+\sigma(v)$ is constant for each edge $u v \in E(G)$. Consequently, we have

$$
M S_{1}(G) \leq \sqrt{m\left(M S D^{\frac{3}{2}}(G)+2 M S_{2}(G)\right)}
$$

Let $G$ be a connected graph with $n$ vertices. Let us define the topological invariant $\Phi(G)$ as follows

$$
\Phi(G)=\frac{\left(\sum_{u \in V(G)} \sigma(u)\right)^{2}}{n \sum_{u \in V(G)} \sigma^{2}(u)}=\frac{4 W(G)^{2}}{n M S^{1}(G)} .
$$

The following theorem shows that $\Phi(G)$ quantifies the degree of transmission regularity of a connected graph $G$. 
Theorem 2.1. Let $G$ be a connected graph with $n$ vertices. Then $\Phi(G) \leq 1$, with equality if and only if $G$ is transmission regular.

Proof. Using Cauchy-Schwartz inequality, we obtain

$$
\left(\sum_{u \in V(G)} \sigma(u)\right)^{2} \leq n \sum_{u \in V(G)} \sigma(u)^{2},
$$

with equality if and only if $\sigma(u)=\sigma(v)$ for each $u, v \in V(G)$. This completes the proof.

Proposition 2.14. Let $G$ be a connected graph with $n$ vertices and $m$-edges. If $\rho_{D}(G)$ denotes the distance spectral radius of $G$, then

$$
2(n-1)-\frac{2 m}{n} \leq \frac{2 W(G)}{n} \leq \rho_{D}(G) .
$$

The left-hand side equality holds if and only if $\operatorname{diam}(G) \leq 2$. The right-hand side equality holds if and only if $G$ is transmission regular.

Proof. The left-hand side inequality is nothing but Lemma 2.9. From Theorem 2.1 and $\left[2\right.$, Theorem 5.5] one obtains that $\frac{2 W(G)}{n} \leqslant \sqrt{\frac{1}{n} M S^{1}(G)} \leqslant \rho_{D}(G)$, with equality if and only if $G$ is transmission regular.

Let us finish this section with following result showing how $W(G), M S_{1}(G)$, and $\xi^{d}(G)$ relates to each other.

Theorem 2.2. [27] Let $G$ be a connected graph on $n \geqslant 3$ vertices. Then

$$
M S_{1}(G) \leqslant 2 n W(G)-\xi^{d}(G)
$$

with equality if and only if $G \cong P_{4}$, or $G \cong K_{n}-k e$, for $k=0,1, \ldots,\left\lfloor\frac{n}{2}\right\rfloor$, where $k e$ denotes a matching of size $k$.

\section{Vertex And Edge Transitive Graphs}

In this section, following Darafsheh [8,34], we aim to use a method which applies group theory to graph theory. For more details regarding the theory of groups and graph theory one can see [15] and [19], respectively.

Let $\Gamma$ be a group acting on a set $X$. We shall denote the action of $\alpha \in \Gamma$ on $x \in X$ by $x^{\alpha}$. Then $U \subseteq X$ is call an orbit of $\Gamma$ on $X$ if for every $x, y \in U$ there exists $\alpha \in \Gamma$ such that $x^{\alpha}=y$. The action of group $\Gamma$ on $X$ is called transitive if $X$ is itself an orbit of $\Gamma$ on $X$.

Let $G$ be a graph. A bijection $\alpha$ on $V(G)$ is called an automorphism of $G$ if it preserves $E(G)$. In other words, $\alpha$ is an automorphism if for each $u, v \in V(G)$, $e=u v \in E(G)$ if and only if $u^{\alpha} v^{\alpha} \in E(G)$. Let us denote by $\operatorname{Aut}(G)$ the set of all automorphisms of $G$.

It is known that $\operatorname{Aut}(G)$ forms a group under the composition of mappings. This is a subgroup of the symmetric group on $V(G)$. Note that $\operatorname{Aut}(G)$ acts on $V(G)$ 
naturally, i.e., for each $\alpha \in \operatorname{Aut}(G)$ and $v \in V(G)$ the action of $\alpha$ on $v, v^{\alpha}$, is defined as $\alpha(v)$. The action of $\operatorname{Aut}(G)$ on $V(G)$ induces an action on $E(G)$. In fact, for $\alpha \in \operatorname{Aut}(G)$ and $e=u v \in E(G)$, the action of $\alpha$ on $e=u v, e^{\alpha}$, is defined as $u^{\alpha} v^{\alpha}$.

A graph $G$ is called vertex-transitive (edge-transitive) if the action of $\operatorname{Aut}(G)$ on $V(G)(E(G))$ is transitive.

Let $G$ be a graph, $V_{1}, V_{2}, \ldots, V_{t}$ be the orbits of $\operatorname{Aut}(G)$ under its natural action on $V(G)$. Then for each $1 \leq i \leq t$ and for $u, v \in V_{i}, \sigma(u)=\sigma(v)$. In particular, if $G$ is vertex transitive $(t=1)$, then for each $u, v \in V(G), \sigma(u)=\sigma(v)$. Therefore vertextransitive graphs are transmission regular. It is known that any vertex-transitive graph is (vertex degree) regular [19] and transmission regular [8], but note vice versa.

Lemma 3.1. Let $G$ be a connected $k$-transmission regular graph with $n$ vertices and $m$ edges. Then

$$
\begin{aligned}
S J(G) & =\frac{m^{2}}{(m-n+2) \sqrt{2 k}}, \quad G A S(G)=\frac{n}{2}, \quad H S(G)=\frac{m}{k}, \\
J(G) & =\frac{m^{2}}{(m-n+2) k} .
\end{aligned}
$$

Lemma 3.2. Let $G$ be a connected vertex-transitive graph with $n$ vertices and $m$ edges and the valency $r$. Then

$$
\begin{aligned}
S J(G) & =\frac{m^{2} \sqrt{n}}{2(m-n+2) \sqrt{W(G)}}, \quad G A S(G)=\frac{2 W(G)}{n}, \\
H S(G) & =\frac{n m}{2 W(G)}=\frac{n^{2} r}{4 W(G)}, \\
J(G) & =\frac{m^{2} n}{2(m-n+2) W(G)}=\frac{m n^{2} r}{4(m-n+2) W(G)} .
\end{aligned}
$$

Proof. If $G$ is a connected vertex-transitive graph with $n$ vertices and $m$ edges, then $G$ is of valency $r$ ( $r$-regular) and $k$-transmission regular, for some natural numbers $r$ and $k$. It follows that $2 m=n r$ and $2 W(G)=n k$.

Lemma 3.3. Let $G$ be a connected $k$-transmission regular with $n$ vertices and $m$ edges. Then

$$
H S(G) \leq \frac{m}{2(n-1)-\frac{2 m}{n}}
$$

with equality if and only if $\operatorname{diam}(G) \leq 2$.

Proof. Follows from Proposition 2.11 and the fact that for a $k$-transmission regular graph $G$ with $n$ vertices and $m$ edges, $H S(G)=\frac{m}{k}$.

Theorem 3.1. Let $G$ be a connected graph with $n$ vertices and $m$ edges. Let us denote the orbits of the action $\operatorname{Aut}(G)$ on $E(G)$ by $E_{1}, E_{2}, \ldots, E_{l}$. Suppose that for 
each $1 \leq i \leq t, e_{i}=u_{i} v_{i}$ is a fixed edge in the orbit $E_{i}$. Then

$$
\begin{gathered}
H S(G)=\sum_{i=1}^{l} \frac{2\left|E_{i}\right|}{\sigma\left(u_{i}\right)+\sigma\left(v_{i}\right)}, \quad S J(G)=\frac{m}{m-n+2} \sum_{i=1}^{l} \frac{\left|E_{i}\right|}{\sqrt{\sigma\left(u_{i}\right)+\sigma\left(v_{i}\right)}}, \\
G A S(G)=\frac{n}{2 m} \sum_{i=1}^{l} \frac{\left|E_{i}\right| \sqrt{\sigma\left(u_{i}\right) \sigma\left(v_{i}\right)}}{\sigma\left(u_{i}\right)+\sigma\left(v_{i}\right)}, \quad \operatorname{irr}_{\operatorname{Tr}}(G)=\sum_{i=1}^{l}\left|E_{i}\right|\left|\sigma\left(u_{i}\right)-\sigma\left(v_{i}\right)\right|, \\
M S_{1}(G)=\sum_{i=1}^{l}\left|E_{i}\right|\left(\sigma\left(u_{i}\right)+\sigma\left(v_{i}\right)\right), \quad M S_{2}(G)=\sum_{i=1}^{l}\left|E_{i}\right| \sigma\left(u_{i}\right) \sigma\left(v_{i}\right) .
\end{gathered}
$$

Corollary 3.1. Let $G$ be a connected graph with $n$ vertices and $m$ edges. If $G$ is edge-transitive and uv is a fixed edge of $G$, then

$$
\begin{aligned}
H S(G) & =\frac{2 m}{\sigma(u)+\sigma(v)}, \quad S J(G)=\frac{m^{2}}{(m-n+2) \sqrt{\sigma(u)+\sigma(v)}}, \\
G A S(G) & =\frac{n}{2} \frac{\sqrt{\sigma(v) \sigma(v)}}{\sigma(u)+\sigma(v)}, \quad M S_{2}(G)=m \sigma(u) \sigma(v), \\
\operatorname{irr}_{\operatorname{Tr}}(G) & =m|\sigma(u)-\sigma(v)|, \quad Q S_{e}(G)=|\sigma(u)-\sigma(v)|, \\
Q S_{v, e}(G) & =\frac{n}{2}(1+|\sigma(u)-\sigma(v)|), \quad M S_{1}(G)=m(\sigma(u)+\sigma(v)) .
\end{aligned}
$$

Fullerenes are zero-dimensional nanostructures, discovered experimentally in 1985 [30]. Fullerenes $C_{n}$ can be drawn for $n=20$ and for all even $n \geq 24$. They have $n$ carbon atoms, $\frac{3 n}{2}$ bonds, 12 pentagonal and $\frac{n}{2}-10$ hexagonal faces. The most important member of the family of fullerenes is $C_{60}$ [30]. The smallest fullerene is $C_{20}$. It is a well-known fact that among all fullerene graphs only $C_{20}$ and $C_{60}$ (see Figure 3) are vertex-transitive [18]. Since for every vertex of $v \in V\left(C_{20}\right), \sigma(v)=50$ and for every $v \in V\left(C_{60}\right), \sigma(v)=278$, then

$$
\begin{aligned}
S J\left(C_{20}\right) & =7.5, \quad G A S\left(C_{20}\right)=50, \quad H S\left(C_{20}\right)=0.6, \\
J\left(C_{20}\right) & =1.5, \quad S J\left(C_{60}\right)=10.73, \quad G A S\left(C_{60}\right)=278, \\
H S\left(C_{60}\right) & =0.32, \quad J\left(C_{60}\right)=0.9 .
\end{aligned}
$$

A nanostructure called achiral polyhex nanotorus (or toroidal fullerenes of parameter $p$ and length $q$, denoted by $T=T[p, q]$ is depicted in Figure 4 and its 2-dimensional molecular graph is in Figure 5. It is regular of valency 3 and has $p q$ vertices and $\frac{3 p q}{2}$ edges. It follows the following proposition.

\section{Proposition 3.1.}

$$
\begin{aligned}
& S J(T)=\frac{9(p q)^{2} \sqrt{p q}}{8(p q+2) \sqrt{W(T)}}, \quad G A S(T)=\frac{2 W(T)}{p q}, \\
& H S(T)=\frac{3(p q)^{2}}{4 W(T)}, \quad J(T)=\frac{9(p q)^{3}}{8(p q+2) W(T)} .
\end{aligned}
$$




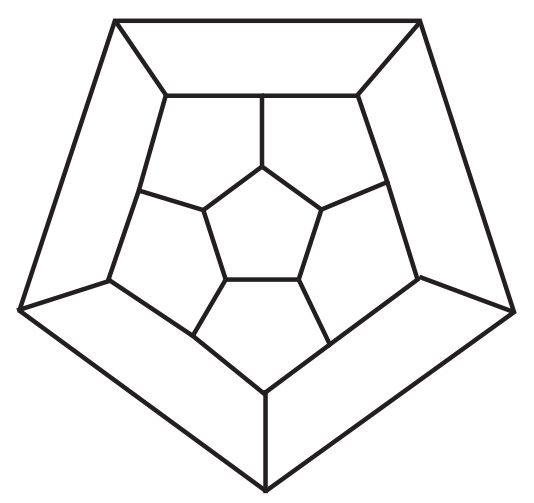

FiguRE 3. 2-dimensional graph of fullerene $C_{20}$

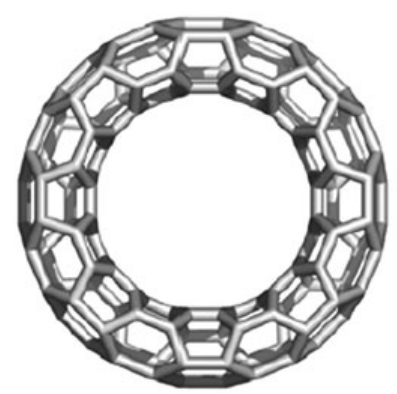

Figure 4. A achiral polyhex nanotorus (or toroidal fullerene) $T[p, q]$

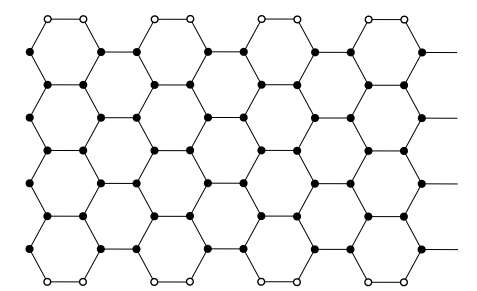

Figure 5. A 2-dimensional lattice for an achiral polyhex nanotorus $T[p, q]$

The vertex set of the hypercube $H_{n}$ consists of all $n$-tuples $\left(b_{1}, b_{2}, \ldots, b_{n}\right)$ with $b_{i} \in\{0,1\}$. Two vertices are adjacent if the corresponding tuples differ in precisely one place. Moreover, $H_{n}$ has exactly $2 n$ vertices and $n 2^{n-1}$ edges.

Lemma 3.4. [8] The hypercube $H_{n}$ is $\left(n 2^{n-1}\right)$-transmission regular which is vertexand edge-transitive.

Therefore from Lemma 3.1 and Lemma 3.4 we have the following result. 


\section{Corollary 3.2.}

$$
\begin{aligned}
S J\left(H_{n}\right) & =\frac{n^{2} 2^{2(n-1)}}{\left(n 2^{n-1}-2 n+2\right) \sqrt{n 2^{n}}}, \quad G A S\left(H_{n}\right)=n, \quad H S\left(H_{n}\right)=2 n^{2} 2^{2(n-1)}, \\
J\left(H_{n}\right) & =\frac{n^{2} 2^{2(n-1)}}{\left(n 2^{n-1}-2 n+2\right) n 2^{n-1}} .
\end{aligned}
$$

\section{On the Application Possibilities of Transmission-Based Topological INDICES IN QSPR STUDIES}

As we have already mentioned, the transmission-based topological indices represent a particular family of distance-based topological invariants. In what follows we demonstrate in examples the promising applications of TT indices in QSPR studies. In the literature we found some TT indices used successfully for predicting physico-chemical properties of unbranched alkanes. Ren [41] introduced a topological index denoted by $X u$, it is defined for an $n$-vertex connected graph $G$ as follows:

$$
X u(G)=\sqrt{n} \log \left(\frac{\sum_{u \in V(G)} d(u) \sigma^{2}(u)}{\sum_{u \in V(G)} d(u) \sigma(u)}\right) .
$$

Analyzing the mono-parametric correlations with different degree-based and distancebased indices (Randić connectivity index, Balaban's $J$ index), the linear prediction model based on $X u(G)$ index gives the best results.

Shamsipur et al. [42] proposed a family of bond-additive TT topological indices, called as shamsipur indices ( $S h_{1}-S h_{10}$ indices) and used them for prediction of different physico-chemical properties of a large number of alkanes and alkane isomers. In [42] for 379 organic compounds ten different versions of $S h$ indices were calculated and their ability were evaluated in QSPR studies. The resulting regression data were compared with the results based on several known topological indices, and in most cases, betters results were obtained by the $S h_{1}-S h_{10}$ indices. For example, using the $S h_{1}$ index defined as

$$
S h_{1}(G)=\log \left(\sum_{u v \in E(G)} \frac{\sigma(u) \sigma(v)}{d(u) d(v)}\right),
$$

a correlation coefficient of 0,983 between the boiling point (BP) and $S h_{1}$ was obtained.

Acknowledgements. The first author would like to thank Persian Gulf University (PGU)'s research council for its support.

\section{REFERENCES}

[1] M. O. Albertson, The irregularity of a graph, Ars Combin. 46 (1997), 219-225. 
[2] M. Aouchiche and P. Hansen, Distance spectra of graphs: a survey, Linear Algebra Appl. 458 (2014), 301-386.

[3] M. Aouchiche and P. Hansen, On a conjecture about Szeged index, European J. Combin. 31 (2010), 1662-1666.

[4] A. T. Balaban, Highly discriminating distance based numerical descriptor, Chemical Physics Letters 89 (1982), 399-404.

[5] A. T. Balaban, Topological indices based on topological distances in molecular graphs, Pure and Applied Chemistry 55 (1983), 199-206.

[6] A. T. Balaban, P. V. Khadikar and S. Aziz, Comparison of topological indices based on iterated sum versus product operations, Iranian Journal of Mathematical Chemistry 1 (2010), 43-67.

[7] F. K. Bell, A note on the irregularity of graphs, Linear Algebra Appl. 161 (1992), 45-54.

[8] M. R. Darafsheh, Computation of topological indices of some graphs, Acta Appl. Math. 110 (2010), 1225-1235.

[9] K. C. Das and I. Gutman, Estimating the Wiener index by means of number of vertices, number of edges, and diameter, MATCH Commun. Math. Comput. Chem. 64 (2010), 647-660.

[10] H. Deng, On the sum-Balaban index, MATCH Commun. Math. Comput. Chem. 66 (2011), $273-284$.

[11] A. Dobrynin and A. A. Kochetova, Degree distance of a graph: a degree analogue of the Wiener index, Journal of Chemical Information and Computer Sciences 34 (1994), 1082-1086.

[12] A. Dobrynin and I. Gutman, On a graph invariant related to the sum of all distances in a graph, Publ. Inst. Math. (Beograd) (N.S.) 56 (1994), 18-22.

[13] A. Dobrynin, R. Entringer and I. Gutman, Wiener index of trees: theory and applications, Acta Appl. Math. 66 (2001), 200-249.

[14] J. K. Doyle and J. E. Graver, Mean distance in a graph, Discrete Math. 17 (1977), 147-154.

[15] J. D. Dixon and B. Mortimer, Permutation Groups, Springer-Verlag, New York, 1996.

[16] R. C. Entringer, D. E. Jackson and D. A. Snyder, Distance in graphs, Czechoslovak Math. J. 26 (1976), 283-296.

[17] G. H. Fath-Tabar, B. Furtula and I. Gutman, A new geometric-arithmetic index, J. Math. Chem. 47 (2014), 477-468.

[18] M. Ghorbani, Remarks on the Balaban index, Serdica J. Comput. 7 (2013), 25-34.

[19] C. Godsil, G. Royle, Algebraic Graph Theory, Springer-Verlag New York, 2001.

[20] S. Gupta, M. Singh and A. K. Madan, Eccentric distance sum: a novel graph invariant for predicting biological and physical properties, J. Math. Anal. Appl. 275 (2002), 386-401.

[21] I. Gutman and Y-N. Yeh, The sum of all distances in bipartite graphs, Math. Slovaca 45 (1995), $327-334$.

[22] I. Gutman and K. C. Das, The first Zagreb index 30 years after, MATCH Commun. Math. Comput. Chem. 50 (2004), 83-92.

[23] I. Gutman, Degree-based topological indices, Croatica Chemica Acta 86 (2013), 351-361.

[24] I. Gutman, Selected properties of the Schultz molecular topological index, Journal of Chemical Information and Computer Sciences 34 (1994), 1087-1089.

[25] A. Ilić and D. Stevanović, On comparing Zagreb indices, MATCH Commun. Math. Comput. Chem. 62 (2009), 681-687.

[26] A. Ilić, S. Klavžar and M. Milanović, On distance-balanced graphs, European J. Combin. 31 (2010), 733-737.

[27] A. Ilić, G. Yub and L. Fengc, On the eccentric distance sum of graphs, J. Math. Anal. Appl. 381 (2011), 590-600.

[28] M. H. Khalifeh, H. Yousefi-Azari, A. R. Ashrafi and S. G. Wagner, Some new results on degreebased graph invariants, European J. Combin. 30 (2009), 1149-1163.

[29] S. Klavžar, A. Rajapakse and I. Gutman, The Szeged and the Wiener index of graphs, Appl. Math. Lett. 9 (1996), 45-49. 
[30] H. W. Kroto, J. R. Heath, S. C. O'Brien, R. F. Curl and R. E. Smalley, C60:Buckminsterfullerene, Nature 318 (1985), 162-163.

[31] B. Liu and Z. You, A survey on comparing Zagreb indices, MATCH Commun. Math. Comput. Chem. 65 (2011), 581-593.

[32] H. Liu and X. F. Pan, On the Wiener index of trees with fixed diameter, MATCH Commun. Math. Comput. Chem. 60 (2008), 85-94.

[33] A. Miličević and S. Nikolić, On variable Zagreb indices, Croatica Chemica Acta 77 (2004), 97-101.

[34] R. Mohammadyari and M. R. Darafsheh, Topological indices of the Kneser graph $K_{n ; k}$, Filomat 26 (2012), 665-672.

[35] M. J. Nadjafi-Arani, H. Khodashenas and A. R. Ashrafi, On the differences between Szeged and Wiener indices of graphs, Discrete Math. 311 (2011), 2233-2237.

[36] S. Nikolić, G. Kovačević, M. Miličević and N. Trinajstić, The Zagreb indices 30 years after, Croatica Chemica Acta 76 (2003), 113-124.

[37] V. Nikiforov, Eigenvalues and degree deviation in graphs, Linear Algebra Appl. 414 (2006), $347-360$.

[38] T. Pisanski and M. Randić, Use of Szeged index and the revised Szeged index for measuring the network bipartivity, Discrete Appl. Math. 158 (2010), 1936-1944.

[39] H. S. Ramane and A. S. Yalnaik, Status connectivity indices of graphs and its applications to the boiling point of benzenoid hydrocarbons, J. Appl. Math. Comput. 1-2 (2017), DOI: $10.1007 / \mathrm{s} 12190-016-1052-5$.

[40] M. Randić, On characterization of molecular branching, Journal of the American Chemical Society 97 (1975), 6609-6615.

[41] B. Ren, A new topological index for QSPR of alcanes, Journal of Chemical Information and Computer Sciences 39 (1999), 139-143.

[42] M. Shamsipur, B. Hemmateenejad and M. Akhound, Highly correlating distance connectivitybased topoligical indices. 1: QSPR studies of alkanes, Bull. Korean Chem. Soc. 25 (2004), 253-259.

[43] A. I. Tomescu, Unicyclic and bicyclic graphs having minimum degree distance, Discrete Appl. Math. 156 (2008), 125-130.

[44] D. B. West, Introduction to Graph Theory, Prentic-Hall, Upper Saddle River, New Jersey, 1996.

[45] D. Vukičević and B. Furtula, Topological index based on the ratios of geometrical and arithmetical means of end-vertex degrees of edges, J. Math. Chem. 46 (2009), 1369-1376.

[46] B. A. Xavier, E. Suresh and I. Gutman, Counting relations for general Zagreb indices, Kragujevac J. Math. 38 (2014), 95-103.

[47] R. Xing and B. Zhou, On the revised Szeged index, Discrete Appl. Math. 159 (2011), 69-78.

[48] B. Zhou and N. Trinajstić, On general sum-connectivity index, J. Math. Chem. 47 (2010), 2010-2018.

[49] L. Zhong, The harmonic index for graphs, Appl. Math. Lett. 25 (2012), 561-566.

[50] L. Zhong and $\mathrm{K} . \mathrm{Xu}$, Inequalities between vertex-degree-based topological indices, MATCH Commun. Math. Comput. Chem. 71 (2014), 627-642.

[51] H. Hosoya, Topological index. A newly proposed quantity characterizing the topological nature of structural isomers of saturated hydrocarbons, Bulletin of the Chemical Society of Japan 44 (1971), 2332-2339. 


\author{
${ }^{1}$ Department of Mathematics, \\ Persian Gulf University, \\ BUSHEHR 7516913817, IRAN \\ Email address: sharafidi@pgu.ac.ir \\ ${ }^{2}$ ÓBUdA UNIVERSITY \\ BÉCsiút 96/B, H-1034 Budapest, Hungary \\ Email address: reti.tamas@bgk. uni-obuda.hu
}

